\title{
Poder y exclusiones formativas en Ciencias de la Salud. Propuestas de mejora en la atención al paciente desde la complementariedad
}

Power and exclusions in Health Sciences training. Suggestions for improvement in patient care from complementarity

\section{Poder e formação exclusões em Ciências da Saúde. Sugestões de melhoria na assistência ao paciente de complementaridade}

David Conde Caballero ${ }^{1}$

${ }^{1}$ Licenciado en Antropología Social y cultural. Diplomado en Enfermería. Universidad de Extremadura

Cómo citar este artículo en edición digital: Conde Caballero, D. (2014) Poder y exclusiones formativas en Ciencias de la Salud. Propuestas de mejora en la atención al paciente desde la complementariedad. Cultura de los Cuidados. (Edición digital) 18, 40. Disponible en: <http://dx.doi.org/10.7184/cuid.2014.40.9>

Correspondencia: C/ Caleros 62. 10002. Cáceres. Correo electrónico: dcondecab@gmail.com Recibido: 09/06/2014; Aceptado: 14/09/2014

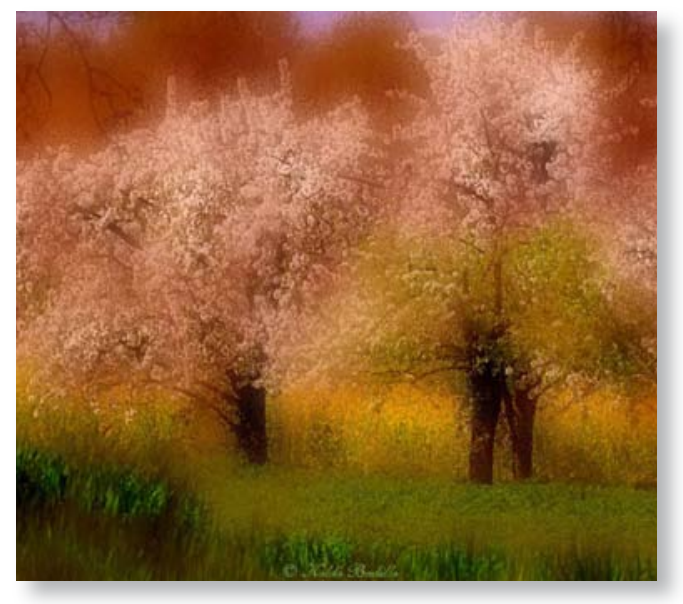

ABSTRACT

Introduction: The importance of the health-disease-care is a universal fact executed through different models. West proposes the Biomedical model, whose achievements are limited by the exclusion that makes the sociocultural variables of the sick.

Objective: A reflection on the possibilities for improvement to enable a close to holism decisive assistance, not only for greater professionalism but also the formation of a real nurse identity, is the main objective.
Methodology: To try to give, long referenced in the field of medical anthropology we venture robust response to the reading of two classics from the likes of Foucault and Gramsci, allowing us to suggest a line in the search for reasons and solutions on the problems surrounds us.

Development: A reasonable way is looming on the contribution that complementary forms of care possible. In this sense, the current nursing should seek a return to its ethos of care, thus moving away from deterministic rules that have condemned the subaltern.

Conclusions: A change in training patterns are established as key feature to determine real improvement in processes of care and allow the basic right to quality care.

Keywords: Healthl, Anthropology, Biomedicine, power, hegemony, complementary.

\section{RESUMO}

Introdução: A importância da saúde-doença-cuidado da saúde é um fato universal executado através de diferentes modelos. Ocidente propõe o modelo biomédico, cujas conquistas 
são limitados pela exclusão que faz com que as variáveis sócio-culturais do doente.

Objetivo: Uma reflexão sobre as possibilidades de melhoria para permitir um fim à assistência decisiva holismo, não só para um maior profissionalismo, mas também a formação de uma identidade enfermeira real, é o objetivo principal.

Metodologia: Para tentar dar uma, muito referenciado no campo da antropologia médica nos aventuramos resposta robusta para a leitura de dois clássicos de nomes como Foucault e Gramsci, o que nos permite sugerir uma linha na busca de razões e soluções sobre os problemas nos rodeia.

Desenvolvimento: Uma maneira razoável é iminente sobre a contribuição que as formas complementares de cuidado possível. Neste sentido, a enfermagem atual deve buscar um retorno ao seu ethos do cuidado, afastando-se assim das regras deterministas que condenaram o subalterno.

Conclusões: A mudança nos padrões de formação são estabelecidos como característica fundamental para determinar real melhoria nos processos de atendimento e permitir que o direito básico de cuidados de qualidade.

Palavras-chave: Saúde, Antropologia, Biomedicina, poder, hegemonia, complementar.

\section{RESUMEN}

Introducción: La importancia de los procesos de salud-enfermedad-atención es un hecho universal ejecutado a través de diferentes modelos. Occidente propone el modelo Biomédico, cuyos logros se ven limitados por la exclusión que realiza a las variables socioculturales del enfermar.

Objetivo: Una reflexión sobre las posibilidades de mejora que permitan una asistencia cercana al holismo, determinante no sólo de una mayor profesionalidad sino también de la conformación de una auténtica identidad enfermera, constituye el objetivo esencial.

Metodología: Para intentar dar una respuesta sólida nos aventuraremos a la lectura de dos autores clásicos de la talla de Foucault y Gramsci, largamente referenciados en el ámbito de la Antropología médica, permitiéndonos sugerir una línea en la búsqueda de motivos y soluciones sobre la problemática que nos envuelve.

Desarrollo: Una vía razonable se vislumbra en el aporte que las formas de atención complementarias posibilitan. En este sentido, la Enfermería actual debe buscar una vuelta a su ethos del cuidado, alejándose así de los preceptos deterministas que la han condenado a la subalternidad.

Conclusiones: Un cambio en los patrones formativos se constituye como eje básico que determine una mejora real en los procesos de atención y permitan el derecho básico de una atención de calidad.

Palabras clave: Salud, Antropología, Biomedicina, poder, hegemonía, complementario.

\section{INTRODUCCIÓN}

No descubriremos nada nuevo en este texto si afirmamos que de todos los elementos que determinan y conforman el entramado estructural básico de una sociedad hay uno que por encima de los demás cobra una especial trascendencia e importancia; tanto por la significación simbólica como por la contribución a la mejora de las condiciones de vida que representa. Hablamos aquí de la preocupación de lo que en el año 1990 Menéndez vino a definir como los procesos de salud-enfermedadatención (Menéndez, 1990); entendidos como todas aquellas formas y modelos de respuesta 
social que luchan en la prevención, protección y curación de los procesos de enfermedad, padecimientos y daños para la salud; los cuales vienen a constituir algunos de los hechos más frecuentes e inevitables que afectan a la vida cotidiana de los conjuntos sociales (Menéndez, 1994) y cuya resolución y erradicación resulta indispensable para asegurar la subsistencia de cualquier conglomerado socio-cultural.

De todas las formas posibles de entender e intentar solucionar los daños provocados en la salud, occidente, como unidad social - que no cultural -, propone desde principios del siglo XIX el modelo conocido bajo el nombre de sistema Biomédico o Alopático, identificado por Menéndez con la denominación de Modelo Médico Hegemónico (MMH) (Menéndez, 2003) por su posición de preeminencia y exclusivismo sobre el resto de las formas de curación/atención; exclusión que ejecuta no sólo en el ámbito ideológico, sino también en el jurídico; todo ello apoyado en una visión de sí mismo en términos de "la única forma correcta de diagnosticar, explicar, atender y solucionar los problemas de la enfermedad, legitimado tanto por criterios científicos como politicos" (Menéndez \& Di Pardo, 1996).

Ante una tan negativa presentación, resulta obligado y pertinente reconocer los métodos y méritos de este modelo en tanto en cuanto su implantación se ha traducido en la mejora de las condiciones y calidad de vida de las poblaciones, hecho éste que resulta difícilmente discutible (Carrió, S. 2006). Es claro que por ejemplo en China, antes de la revolución, podíamos encontrar una de las más altas tasas de mortalidad general y de mortalidad infantil existentes en el planeta pese a tener uno de los más difundidos y codificados sistemas de medicina alternativa (Menéndez, 1981); hecho que sólo vino a paliarse con la instauración de las formas y preceptos de la medicina Alopática.

Sin embargo, el reconocimiento de sus méritos no exime de la necesidad de un análisis maduro de sus particularidades en busca de la mejora constante en los procesos de atención sobre las personas que lo precisan, dado que en él no sólo abundan bondades, sino que nos encontramos con una visión biologicista, reduccionista, individualista y puramente racionalista que viene a determinar un proceso de aplicación sanitaria de corte netamente cientifista; donde el uso de la tecnología, la sobreexposición farmacológica y el uso de protocolos e índices se asocian a buenos resultados terapéuticos; lo que nos obliga a mantener como mínimo una actitud crítica por parte de los profesionales de la sanidad, entre ellos la Enfermería. En este sentido, merece la pena hacerse eco de los pensamientos contrarios que surgen respecto del papel de la medicina Alopática en su forma actual y las posibilidades de mejora que se atisbarían desde la complementariedad. Del mismo modo, jugoso es el debate acerca de las reticencias al cambio, resultando especialmente reseñables las palabras de clásicos de la talla de Foucault o Gramsci, o más recientemente las miradas ácidas que desde el ámbito de la Antropología médica han efectuado autores de la talla de Menéndez, Good o Kleinman; miradas que trataremos de englobar y relacionar en este texto, encontrando, o al menos intentando encontrar, los posibles motivos y resistencias que impiden cambios en apariencia tautológicos, pero que sin embargo se encuentran con poderosas trabas estamentales ejecutadas soslayadamente desde el ámbito institucional y burocrático.

\section{OBJETIVOS}

La Biomedicina en general y la Enfermería en particular, en una búsqueda de excelencia 
en su atención sobre el paciente, debieran haber sido capaces de comprender a estas alturas -no sólo en el ámbito académico, sino con su aplicación en al campo asistencial- que es necesario un acercamiento a la manera específica en que cada individuo experimenta su enfermedad, lo que tendría, sin lugar a dudas, una incidencia definitiva sobre el tratamiento y el proceso curativo, dado que los pacientes valoran de forma muy positiva el hecho de ser escuchados, entendidos y concebidos como sujetos culturales e históricos (Cortés Duque, Uribe, \& Vásquez, 2005). En el caso particular de la Enfermería, el entrenamiento y posterior uso de algunas formas complementarias ha mostrado el camino de esa excelsitud posibilitando la vuelta a su propio ethos primigenio con foco principal en las necesidades y cuidados del paciente, permitiéndole alejarse así del positivismo sin paliativos al que se ha visto abocada por su tradicional seguimiento de los paradigmas médicos que la han distanciado de los sufrimientos, significados y contradicciones personales y orientado a lo que se denominó como práctica enfermera basada en la "evidencia" (Juárez, 2013) y su "alter ego", la metodología del Proceso de Atención Enfermero.

La reflexión sobre las posibilidades de mejora desde esa complementariedad -y los motivos de su falta de aplicación- que permitan una asistencia cercana al holismo del cuidado, determinante no sólo de una mayor profesionalidad sino también de la conformación de una auténtica identidad enfermera dentro del paradigma biomédico, constituye el objetivo esencial de este escrito; que pretende no sólo cavilar desde la autoría, sino hacer pensar a los profesionales que día a día prestan sus servicios sobre personas deseosas de otro tipo de atención, de otra forma de realidad asistencial que los contemple como parte y no como objeto del proceso y que en consecuencia se traduzca en una necesaria mejora en los sistemas de salud postmodernos.

\section{METODOLOGÍA}

Para intentar dar una respuesta sólida a las cuestiones planteadas nos aventuraremos aquí la compleja tarea de recurrir a la lectura relacional y comparativa de dos autores clásicos de la talla de Foucault y Gramsci, largamente referenciados en el ámbito de la Antropología médica y que nos permitirán articular y estructurar con base teórica la casuística aquí expuesta. Foucault, que a pesar de su peso e importancia en el análisis de las realidades sanitarias contemporáneas raramente entra a formar parte del conocimiento formativo universitario de los profesionales del sector, resultando sólo posible su estudio en la mayoría de las ocasiones a través de ciclos de formación de post-graduación (Amezcua, 2009); se constituye como autor básico que nos permite entender las estructuras de poder actuales a través de su innegable capacidad para la "arqueología del presente". En su análisis del poder, al que Nietzsche se refiere como algo innato al ser humano (Baeza, 2004), y la institucionalización del mismo, llegó a afirmar que "la medicina se impone al individuo, enfermo o no, como acto de autoridad" (Foucault, 1976); definiendo el concepto de autoridad médica dentro de las relaciones de poder en el entramado de la sociedad y determinando que la relación médico-paciente implica en todo momento una relación de poder-sumisión.

El estudio de un concepto de la transcendencia que tiene el poder quedaría huérfano sí el mismo no se articula en su relación con la noción de hegemonía. En este empeño, el teórico italiano Gramsci defiende como en las 


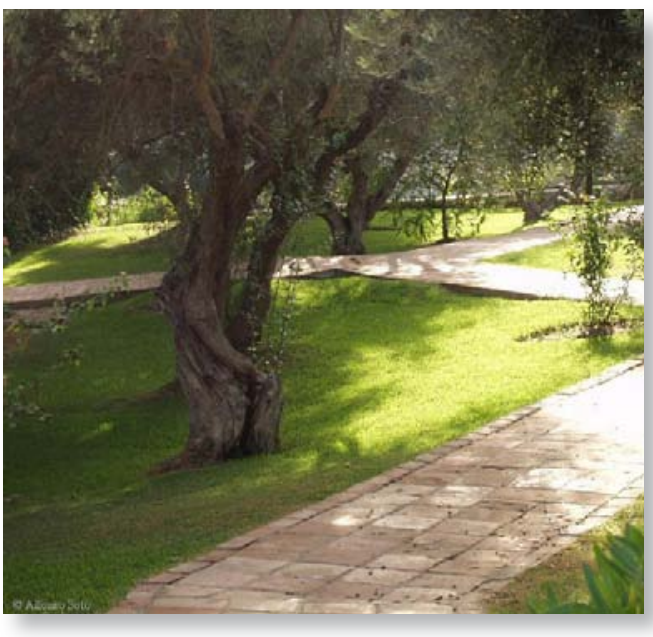

modernas sociedades capitalistas el poder $\mathrm{y}$ la autoridad en el sentido de Foucault vienen determinados en su esencia por la existencia de una hegemonía (Mercado López, 2003). En consecuencia, los métodos alternativos al status establecido no serán vistos más que como tentativas de re-estructuración de las relaciones sociales en clave de hegemonía y posicionamiento social, entendiendo la misma como capacidad de dirección intelectual y moral, y ejecutada por las clases dominantes entre otros aspectos a través de las instituciones educativas. Una aproximación a la obra de Gramsci se encumbra como fundamental para la comprensión de la dialéctica hegemónica y del sistema de relaciones de fuerzas que actúan en los procesos de salud-enfermedad (Pizza, 2005), cuya máxima expresión se observa en los procesos asimétricos y subordinados de relación médico-paciente, donde se da una exclusión casi total de los saberes y opiniones del propio interesado.

De este modo, una lectura reflexiva de clásicos de tal envergadura junto a los postulados de la Antropología médica contemporánea, nos permitirán ejecutar una línea reflexiva en la búsqueda de motivos y soluciones sobre la problemática que nos envuelve.

\section{DESARROLLO}

Una buena manera de poder situarnos en la realidad esbozada es recorrer algunas las particularidades negativas biomédicas que afectaran en mayor o menor medida tanto a profesionales como a pacientes implicados en los procesos de salud-enfermedad-atención. Así, cuando hablamos de sistemas médicos Alopáticos o Biomédicos resultan fácilmente reconocibles algunas de las que aquí enunciamos:

- Atención fundamentalmente biologicista; al abrigo de la corriente conocida bajo el nombre de determinismo biológico. La cual viene a plantear una "...compresión de la vida, la patología y la muerte como resultado de causas exclusivamente biológicas, aunque en algún momento pueda abrir la puerta al papel de las contingencias del mundo social, la pobreza, la cultura, los hábitos dietéticos, las desigualdades sociales u otros factores que serán aprehendidos como causas secundarias..." (Martínez Hernáez, 2008).

- Predomino del uso de la tecnología sobre otras formas de atención. En este contexto se somete al paciente a un proceso que en ocasiones se torna como poco humanizado y frío que convierte al individuo en un elemento aislado y descontextualizado de los aspectos del vivir, alejando a disciplinas como la Enfermería de su primigenia razón de ser.

- Profesionales sanitarios hiper-especializados que brindan una atención sumamente fragmentada; dejando de este modo poco lugar para una concepción holística del proceso de atención. Como indica Hanh: "la patología Biomédica contemporánea diseña su progreso en términos de unidades cada vez más pequeñas" (Hahn, 1995). La noción de patología moderna se aleja cada vez más de su significado etimológico de pathos o sufrimiento para 
recortar, de forma cada vez más atomizada, las unidades que componen el cuerpo biológico (Martínez Hernáez, 2008).

- Centrado en la enfermedad del individuo y no en el propio ser. El modelo tiende a ver al paciente como un mero conjunto de signos y síntomas más que como un individuo biopsico-social; atropellando en consecuencia las realidades socio-culturales de los pacientes al universalizar sus padecimientos y negando en consecuencia que lo cultural o lo psicológico pueda afectar al orden de lo biológico.

- Se trata esta de una forma de atención sanitaria determinada por un hiperbólico consumo de sustancias medicamentosas de síntesis química favorecido y potenciado por la industria químico/farmacéutica cuyos largos tentáculos se introducen soslayadamente sobre los procesos de atención médica, determinando líneas de medicalización ya no sólo de los padeceres, sino también de algunos comportamientos que lejos de ser patológicos forman parte de la normalidad de la vida y que son sometidos contra natura al uso de medicación, donde todo es posible de ser considerado como "enfermo" y en consecuencia debe ser "normalizado" (Menéndez, 2003). La Biomedicina como instrumento del capitalismo (Martínez Hernáez, 2008).

- Existen otras características que podrían complementar a las ya mencionadas como son el reduccionismo, la a-historicidad, la visión de la salud como mercancía a consecuencia de la influencia de los procesos post-modernistas, la eficacia pragmática o la división dualista del universo de herencia cristiana y cartesiana que divide continuamente entre naturaleza y cultura, ciencia dura y blanda o cuerpo y mente, a pesar de que la evidencia de la existencia de las enfermedades psicosomáticas o de trastornos de somatización viene a rebatir en la prác- tica médica diaria esta forma de entender los procesos de morbilidad.

En esta compleja realidad, deberíamos escudriñar en el tiempo para poder aventurarnos a vislumbrar algunos de los motivos que explicarían esa visión en exceso positivista, experimental y fragmentada con la que este modelo se enfrenta a los procesos de salud/ enfermedad. Algunas de las características esenciales del modelo Alopático han sido relacionadas por diferentes autores con la necesidad que las ciencias médicas han tenido históricamente de despojarse del oscurantismo de una época donde la concepción mágico-religiosa determinaba el paradigma del conocimiento galénico. En contraposición, nace una forma de atención sanitaria donde el método científico se erige como su principal seña de identidad creando un potente entramado que permitiría examinar, clasificar y tratar enfermedades de forma casi automatizada. Así, el mundo se divide en sanos y enfermos; siendo la enfermedad aquello que el Médico pueda reconocer, demostrar y clasificar por procedimientos previamente predeterminados; dejando como consecuencia sin cuartel a las variables humanas, sociales o culturales del enfermar y reduciendo los procesos mórbidos a variables estrictamente biológicas y por lo tanto objetivables, cultivo que determinará una perspectiva distorsionada de la atención sobre el paciente. Conjugados, se dará el caldo de cultivo para la creación y explotación de lo que Mishler definió como "una subcultura con sus creencias institucionalizadas, valores y prácticas" (Martínez Hernáez, 2008).

Así las cosas, con una corpus de conocimiento alrededor de la salud más preocupado de curar enfermedades que de atender enfermos -incluyendo, paradójicamente, a la Enfermería-, resulta casi obligado el surgimiento 
de voces cada vez más contrariadas que cuestionen y reclamen el desarrollo de formas de atención que parcialmente puedan complementar a la Biomedicina en la mejora de sus defectos; apostando por la apertura del abanico terapéutico hacia otras formas de entender la atención. Al amparo de esta nueva forma de concebir la realidad surgen, con una fuerza cada vez más evidente, las denominadas como formas terapéuticas complementarias y alternativas; espoleadas no sólo por el uso creciente de las mismas por parte de la población, sino al mismo tiempo apoyadas institucionalmente por la máxima esfera sanitaria mundial, la OMS, que favorece y potencia el uso de este tipo de prácticas por haber demostrado con creces su utilidad sobre el paciente con un riesgo mínimo para la salud (OMS, 2002) y reconociendo su capacidad para complementar y por tanto mejorar las capacidades y actuaciones realizadas por el sistema Alopático; aportando, entre otras muchas cuestiones, que el punto de vista del paciente no supone un estorbo o una limitación en el tiempo de atención que se le asigna a cada uno de los usuarios y que además no sólo permite mejorar la atención, sino la humanidad sobre dicho proceso al tener una visión mucho más holista del paciente, alcanzado por fin esa tan poco lograda definición de individuo bio-psico-social.

En un esfuerzo de síntesis y conjugación que permita contextualizar al lector, desgraciadamente poco acostumbrado a estas "otras formas", tras una revisión de las diferentes opciones bibliográficas más importantes en la materia, concluimos la división que aquí se propone y ya propuesta por Conde (Conde, 2011): en consecuencia definimos la medicina alternativa como aquel sistema o forma de cuidado que pretende ser una alternativa total al sistema Biomédico. Es decir, constituye o pretende sustituir al modelo sanitario Alopático y para ello se basa no sólo en un uso tradicional ampliamente establecido y una dilatada experiencia, sino en una institucionalización importante y en la existencia de una ciencia perfectamente documentada. Los ejemplos más conocidos son el sistema médico chino, los sistemas nativos de indígenas americanos o el sistema Ayurvédico Hindú.

Teniendo clara esta primera definición, ponemos un especial énfasis en la diferenciación existente entre las formas de conocimiento mencionadas y lo que conocemos como terapias complementarias. Con este término nos referimos a una gran variedad de formas preventivas y terapéuticas que se encuentran fuera de lo académicamente establecido por el sistema Biomédico pero que sin lugar a dudas pueden complementar su actuación mejorando la atención y el servicio que se presta al usuario. En los últimos años este tipo de acciones han experimentado un aumento espectacular en su demanda y uso, hasta tal punto que muchos profesionales de la salud se han especializado en las mismas post-graduación con el objetivo de mejorar la atención; a pesar de las reticencias que algunos profesionales muestran al respecto con conservadurismos doctos poco adaptados a las necesidades y demandas reales de los usuarios de servicios sanitarios (Conde, 2011). En cualquier caso, lo que nos interesa aquí es el concepto de complementariedad, puesto que aunque la mayoría de ellas se tratan de ramas en la actualidad muy bien documentadas, por lo general se refieren a actuaciones concretas sobre ciertos problemas determinados. Este tipo de terapias podrían aportarnos, dentro del variado universo de opciones que representan, la posibilidad de que el foco gire hacia el enfermo y no hacia las enfermedades; o la posibilidad de efectuar una interpretación 
holística que tome en consideración la importancia del mundo fenomenológico del paciente en los procesos de salud-enfermedad, evitando así que los profesionales sanitarios se conviertan en "técnicos de cuerpos enfermos"(Carrió, S $\&$ De Cunto, 2008) más que en profesionales de la salud.

Existe una amplia y compleja variedad de formas complementarias con capacidad para utilizarse según en qué fase del proceso tanto mórbido como preventivo nos encontremos $y$ cuyo uso suscita reacciones que se mueven en un amplio abanico que va desde un profundo escepticismo basado en la falta de información, hasta un entusiasmo de uso desmedido carente de toda crítica e igualmente poco racional y censurable. Por citar tan solo algunas, pasarían por las terapias físicas como la quiropráctica, masajes o yoga; las terapias termales; la homeopatía; la quiropráctica; la reflexología; técnicas de relajación como la meditación, la visualización, terapias artísticas, psicoterapias individuales, la narrativa, musicoterapias, hipnosis; hierbas y otros remedios de carácter natural; medicina tradicional herbácea. fitoterapia; y un largo etcétera de posibilidades que en diferentes momentos y situaciones terapéutico/preventivas pueden ser aplicadas por los diferentes profesionales de la salud implicados en el proceso; todas ellas englobadas por Menéndez en lo que define como Modelo Médico Alternativo (Menéndez, 1981), cuyo eje de agrupación estaría determinado porque presentan concepciones globalizadoras sociosimbólicas de los padecimientos y tendencia a la no medicalización por medio de elementos de síntesis química; justo, la solución a uno de los principales elementos negativos de la Biomedicina.

Insistimos una vez más, dada la transcendencia del razonamiento, en la necesidad de

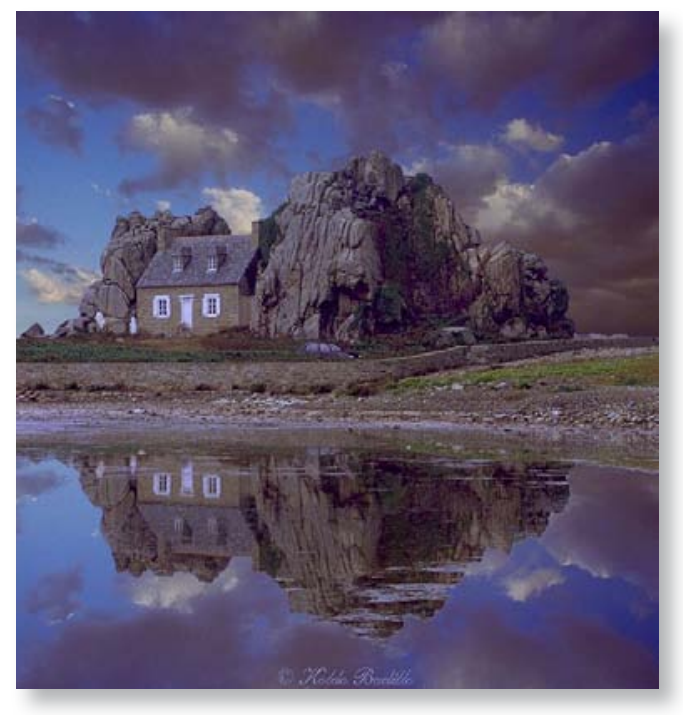

no considerar las diferentes opciones, modelo Biomédico y terapias complementarias, como estáticas y aisladas cada una en sí mismas. Sino que desde estas líneas enfatizamos y recomendamos la existencia de un proceso dinámico de intercambio de información y conocimientos en el que se fomente no el uso individual, sino el uso relacional entre dos o más formas de atención dentro de un proceso de normalidad terapéutica sobre la persona con necesidades. Como bien dicen Nesse y Williams en su obra "Why we get Sick ?" (“Por qué enfermamos?”) :

"...No se pretende pues impulsar aquí una alternativa a la práctica médica actual, sino más bien, una perspectiva adicional desde un corpus de conocimientos médicos que han sido totalmente olvidados por el estamento médico..."

Parece claro, a la luz de lo aquí esgrimido, que la única forma de alcanzar la excelencia propuesta pasa por el giro de las concepciones Biomédicas hacia modelos epistemológicos biopsicosociales de salud que superen de una vez por todas los preceptos explicativos unicausalísticos del enfermar por un enfoque de multideterminación y multicausalidad (Madariaga, 2008); validándose plenamente las 
formas de saber que ofrecen las formas complementarias a través del entendimiento de la atención sanitaria alejada de la verticalidad que supone la figura del Médico en la cúspide del proceso y el resto de profesionales en su base, para aproximarse por el contrario a la horizontalidad que determina la colaboración y aceptación de otras formas de atención con componentes biológicos, psicológicos y sociales. En este sentido, sólo la Psiquiatría parece haber aceptado con claridad la nueva realidad que se demanda desde la calle, siendo hasta el momento la única forma de Biomedicina donde por ejemplo se acepta el hablar y el ser escuchado como arma terapéutica (Carrio, Silvia, 2006) e identificando como una opción válida el componente social y psíquico de la salud.

Sí lo hasta el momento expuesto resulta acertado en su planteamiento, se hace imperativo plantear cuestiones desde la reflexividad: ¿cuáles serían las razones para explicar la negación a tamaña evidencia en el uso diario de la práctica sanitaria con sus consiguientes posibilidades de mejora? ¿Cuál es el camino a seguir para lograr un cambio en este sentido?:

Reticencias al cambio. Poder y hegemonía en torno a la salud.

Para encontrar respuesta a la primera de las cuestiones recurriremos al concepto de poder, y en concreto al concepto de poder médico. El poder social del Médico se legitima de la siguiente manera: la sociedad, en su conjunto, entiende que pocas cosas resultan tan importantes como el mantenimiento y/o la recuperación de su estado de salud. Consecuencia de ello tiene una serie de demandas transcendentales hacia la profesión médica que, a cambio de comprometerse a atenderlas de forma adecuada mediante su capacidad de adentrarse en la "compleja maquinaria humana", le garantiza una serie de privilegios en el orden social en forma de estatus y prestigio en lo intangible $y$ simbólico, pero también le consiente un posicionamiento de clase dominante en lo económico, ideológico y político, con su participación en la toma de ciertas decisiones en lo que podría ser visto como un "pacto o contrato social"; que lejos de ser casual ha ido adquiriendo cuerpo a lo largo de la historia mediante una sólida "autoridad cultural" traducida en una enorme capacidad de convencimiento social de que su actividad es esencial y vital para el bien de la comunidad. El estamento médico no sólo es, por así decirlo, un guardián de lo más preciado, la vida (Pierantoni \& Machado, 1994), sino que desde una visión funcionalista en el sentido esgrimido por Parsons se trata del marco institucional donde acabar con la desviación que supone la enfermedad, así como el encargado de establecer el retorno del paciente a las obligaciones sociales mediante la terapia (Martínez Hernáez, 2008). De esta forma se intercambiaría obediencia por seguridad.

Una lectura comparada, relacional y reflexiva tanto de Foucault como de Gramsci, nos ofrece una perspectiva desde la que intentar abordar la negación del cambio en el modelo Biomédico y sus innegables consecuencias sobre el paciente y sobre el resto de profesiones sanitarias. Estructurándose esencialmente desde el juego de fuerzas y reticencias del estamento médico como grupo social que necesita imponer su hegemonía mediante su proclamación como referencia científica e ideológica necesaria y legitimada de las acciones contra la enfermedad, creando de este modo en su beneficio un modelo de atención sanitaria jerárquico, asimétrico y clasista (Menéndez, 1981) que determinan en consecuencia que las formas complementarias y/o alternativas de atención, así como el resto de los profesionales sanitarios, pasen a cobrar un rol de subalternidad intencionada. No existe he- 
gemonía sin subalternidad o más bien, existe la hegemonía porque existe la subalternidad.

En esta línea, la horizontalidad o el trabajo coordinado y retroalimentado de los actores médicos con las terapias complementarias que podrían ser aplicadas no sólo por profesionales médicos, sino por otros sanitarios con la formación adecuada como son Enfermeras/ os, Podólogos/as, Fisioterapeutas o Terapeutas Ocupacionales... se interpretaría como una igualación posicional y la consiguiente pérdida hegemónica que bien podría venir a poner en duda el rol superlativo del estamento médico en el ideario colectivo social de los cuidados de la salud y su consecuencia directa en forma de pérdida de poder y posición social. Situación que como es lógico resultaría incomoda; por lo que se constituye como elemento determinante para que la transformación de la perspectiva Biomédica hacia otra de carácter Bio-psicosocial colaborativa se encuentre frenada días desde una ámbito puramente estamental y un aparato enteramente burocrático de freno.

La exclusión por tanto que la epistemología Biomédica -entendiendo aquí por Biomédico al colectivo médico- ejecuta parece no ser por tanto aleatoria. Sino que es ejercida en el plano ideológico para posteriormente extrapolarse a los planos social y político-económico según enuncia Menéndez (Menéndez, 1981) y siguiendo con el racionamiento Foucaultiano según el cual para lograr la persistencia del poder se exige un "enfrentamiento entre fuerzas" donde sólo es posible sí se lo "arrebatamos a los otros" (Foucault, M, 1976). El poder a través del saber en un juego que favorece la verticalidad y el unidireccionalismo.

Atendiendo a la dialéctica Gramsciana, no podemos dejar de hacer mención a aquellas estructuras organizacionales médicas a través de las que se articula el mantenimiento de esta situación de hegemonía/poder y que sería imposible sin su más completa connivencia. La complejidad estructural es demasiado intrincada para ser abordada sobre estas líneas, por lo que simplemente atisbaremos aquellas que a nuestro entender pudieran ser fundamentales. Así ocurre por ejemplo con las Facultades de Medicina y otros Grados universitarios sanitarios, así como los Colegios Profesionales y otras organizaciones en tanto en cuanto influyan mediante la colaboración en la conformación de los planes formativos de las diferentes titulaciones, los cuales, dada la parcialidad con la que se encuentran elaborados, vienen a favorecer una educación universitaria sesgada en la que los futuros profesionales dudan de la eficacia de otras formas de atender al paciente subvalorando subconscientemente sus posibilidades; por lo que en su ideario conforman la creencia de que "su medicina" sigue siendo la "única forma" de medicina posible, e ignorando el esencial papel que juega lo social y lo cultural en los procesos de salud-enfermedad, aunque ello sea contraproducente o imposibilite mejoras claras en los tratamientos que se llevan a cabo. El aprendizaje se hace de esta forma a partir de contenidos meramente biológicos, donde los procesos sociales, culturales y psicológicos son anecdóticos, y donde no hay información sobre otras formas de atención. Como indica el Dr. Hernán Baeza: "algo de lo que hacemos los Médicos no está bien, porque nuestra manera habitual de actuar es sin tomar en cuenta, o sin conciencia, muchos de los problemas de los pacientes, y además, sentimos que es natural que sea así" (Baeza, Hernan, 2004). Así las cosas, los modelos curriculares presentes en las profesiones sanitarias no contemplan, salvo excepciones contadas, una formación adecuada que permita conocer otras 
posibilidades en la atención sobre el paciente. Sí analizamos algunos ejemplos de planes formativos en Universidades españolas caeremos en la cuenta de que en la mayoría de las carreras sanitarias esta opción es casi nula, como en el caso de la Enfermería o la Terapia Ocupacional, para pasar a ser inexistente en el currículum formativo de la Medicina. Como bien formularon MURCIA y JARAMILLO(Murcia \& Jaramillo, 2001):

“... existe la tendencia a formular políticas a partir de un único modelo y diseño, enseñado a lo largo de la historia por los positivistas, cual es la experimentación, y que por ende sólo aquello que pueda ser verificado, comprobado, manipulado, y controlado es considerado como científico. Esta creencia ha hecho, además, que se considere como válido sólo el conocimiento científico que contenga análisis estadístico o matemático, incluso para abordar fenómenos psicológicos, antropológicos y hasta sociales..."

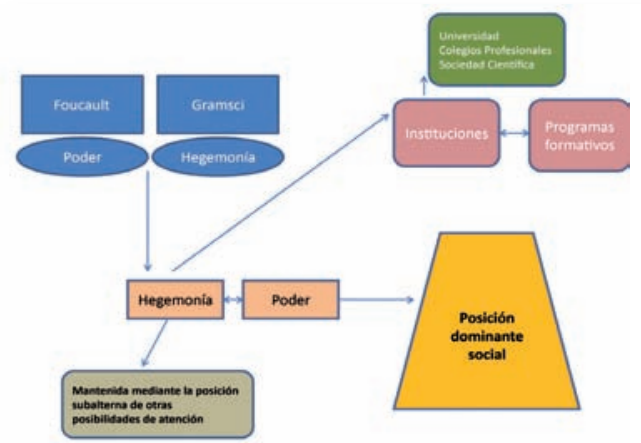

Figura 1. Relación de los conceptos de poder y hegemonía y su papel en la negación de otras posibilidades de atención medica mediante el control de los programas y planes formativos de los estudios universitarios.

\section{Conclusiones post-relacionales.}

Ponemos aquí encima de la mesa, como conclusión y como respuesta a la segunda de las cuestiones planteadas, el innegociable axioma que debe suponer para los profesionales sanitarios la mejora de la calidad asistencial. Requisito indispensable para la consecución de tan loable objetivo resulta la necesidad de una instrucción académica regida por la línea conductora de la diversidad paradigmática que permita a las diferentes opciones preventivo-terapéuticas establecer relaciones de influencia y confianza mutua dentro de la praxis diaria; donde ninguna de la soluciones adquiera carácter de superioridad una respecto de las otras y convivan desde la simetría, otorgando a la asistencia la dimensión de un universo nuevo de posibilidades de atención medica a lo que la OMS se refiere como modelo "integrado de salud" (OMS, 2002), en una escala determinada por el nivel de integración de las terapias complementarias en el sistema médico Alopático y dejando al margen estructuras de poder fáctico establecidas y posicionamientos sociales de hegemonía que redundan en contra de los intereses de las personas que acuden en busca de mejorar su estado de salud.

No se defiende así en este escrito una sustitución de las técnicas Alopáticas por técnicas complementarias o tradicionales, esto sería una quimera falsacionista e irreal; sino que propugnamos y apostamos por una tendencia que establezca lazos intensos de colaboración y complementación basadas en una formación y una institucionalización que, a diferencia de la actual, permita volver a dar importancia a las significaciones del paciente, a su contexto socio-cultural, a otras formas de atención que no entiendan la medicina o formas de curación como un sistema vertical en cuyo vértice dormita el sistema Alopático; sino como un sistema horizontal cuyo objetivo esencial se centra en la mejora de las condiciones sanitarias en la línea de los sistemas bio-psicosociales, tan alabados como faltos de implantación, que aportarían una nueva forma de relación mucho más intensa entre el paciente y el profesional, 
incluyéndose de manera taxativa los significados personales y culturales del afligido.

Un cambio de semejante envergadura en la realidad diaria de los sistemas médicos debiera constituir un desafío cuyo horizonte supusiera la mejora de la atención y en la que sin lugar a dudas debiera jugar un papel de preponderancia la Enfermería, que en una búsqueda de su propio Ethos primigenio en torno al cuidado debe perseguir el enriquecimiento del trato y la atención para con el paciente, no sólo en el ámbito personal, sino sin duda también en el profesional, a través de la búsqueda de otras alternativas, otras miradas, otras visiones, otros caminos circundantes alrededor del holismo sanitario y alejándose de los modelos de evidencia empíricos tan apegados a los protocolos, escalas y hojas de valoración que han determinado una "Enfermería centrada en el profesional de Enfermería” (Valverde, 2008) y menos en el paciente.

La conquista de la salud y su mejora continua como derecho de la ciudadanía debe irremediablemente estar contenida en un proyecto social donde la educación y la formación mas adecuada de los futuros profesionales sanitarios debe ocupar un lugar, sin lugar a dudas, de privilegio; en el que la moderna práctica médica en términos positivistas debe asumir la importancia de la cuestión y permitir la complementariedad en las actuaciones sobre el paciente. Alejándose de premisas de poder, hegemonismo y posicionamiento social en pro de los intereses de un determinado estamento sanitario en lugar de centrado en el objetivo indiscutible de la mejora de la calidad asistencial.

\section{BIBLIOGRAFÍA}

Amezcua Martínez, M. (2009) Foucault y las enfermeras. Pulsando el poder de lo cotidiano.

- Revista Índex de Enfermería. 18(2): 77-79.
- Baeza, H. (2004) El poder del Médico. Revista de la SEMG. (60): 13-18.

- Brody, H. (1992) The Healer's Power. New Haven and London. Yale University Press, Yale.

- Carrió, S.(2006) Aproximaciones de la Medicina narrativa. Revista de Educación médica. 6(1): 14-19.

- Carrió, S. (2008) Medicina narrativa: una estrategia para formar profesionales reflexivos Revista Conexión pediátrica. 3: 1-2.

- Carrió, S. \& De Cunto, C. (2008) Medicina narrativa en Pediatría: relato de una experiencia. Arch Argent Pediatric. 106(2): 128-142.

- Conde, D. (2011) Posibilidades de la medicina tradicional hurdana en el entorno sanitario actual de la zona. Creatspace Independent Publishing Platorm. Disponible en: http://www.amazon.es/Posibilidades-medicinatradicional-hurdana-sanitario/dp/1490522743

- Cortés Duque, C; Uribe, C. \& Vásquez, R. (2005) Etnografía Clínica y narrativas de enfermedad de pacientes afectados con trastorno obsesivo-compulsivo; Clinical

- Ethnography and Illness Narratives of Patients Affected with Obsessive-Compulsive

- Disorder. Revista Colombiana de Psiquiatría. 34(2): 190-219.

- Duarte Gómez, M. (2003) Medicina occidental y otras alternativas: ¿es posible su complementariedad? Reflexiones conceptuales. Cadernos de Saúde Pública. 19(2):635-643.

- Fernández, J; Santiago, F; Villaverde, C; Gutiérrez, M. et al (2007) Contenido simbólico de la bata blanca de los médicos. Gazeta de Antropología, 23. Disponible en: https:// www.researchgate.net/publication/28182483_Contenido_simblico_de_la_bata_blanca_de_los_mdicos

- Foucault, M.(1976) La crisis de la medicina o la crisis de la antimedicina. Educacion medica y salud, 10(2).

- Hahn, R. (1995) Sickness and healing: An anthropological perspective. Yale UniversityPress, Yale.

- Madariaga, C. (2008) La crisis del modelo biomédico y los desafíos actuales. Revista Reflexión, (36). Disponible en: http://www.academia.edu/5111749/INTERCULARIDAD_SALUD_Y_DERECHOS_HUMANOS_HACIA_UN_CAMBIO_EPISTEMOL\%C3\%93GICO 
- Mariano, J; Rodríguez, B; Conde, D. (2013). Cuidados basados en narrativas. Redefiniendo la jerarquía de la evidencia. Index de Enfermería (edición digital); 22(12). Disponible en: http://www.index-f.com/index-enfermeria/v22n1-2/7977.php

- Márquez, R. (2010) De las narrativas de la locura: ; yo no estoy loco! ¿Por qué estoy aquí? Revista de recerca i formacio en Antropología, (12):1-25. Disponible en: http:// revista-redes.rediris.es/Periferia/Articles/4-Marquez.pdf

- Martínez Hernáez, A. (2008) Antropología médica. Teorías sobre la cultura, el poder y la enfermedad. Anthropos Editorial, Barcelona.

- Menéndez, E.(1994) La enfermedad y la curación. ¿Qué es la medicina tradicional? Revista alteridades. 4(7): 71-83.

- Menéndez, E. (1981) La crisis del modelo médico y las alternativas autogestionarias en salud. Revista Alteridades. 19(7): 65-90.

- Menéndez, E. (2003) Modelos de atención de los padecimientos: de exclusiones teóricas y articulaciones prácticas. Ciênc Saúde Coletiva. 8(1): 185-208.

- Menéndez, E. (1990) Antropología médica. Orientaciones, desigualdades e transacciones. Ediciones de la Casa Chata. México:

- Mercado López, S. (2003) Estructura y relación de poder en los prospectos de medicamentos vendidos en España. Estudios filológicos. (38): 93-110.
- Murcia, N. \& Jaramillo, L. (2001) La complementariedad como posibilidad en la estructuración de diseños de investigación cualitativa. Revista Índex de Enfermería. 35:29-33. Disponible en: http://www.index-f.com/ index-enfermeria/35revista/35_articulo_29-33.php

- OMS (2002) Pautas generales para las metodologías de investigación y evaluación de la medicina tradicional: WHO/EDM/TRM/2000.1. Organización Mundial de la Salud, Ginebra.

- OMS (2002) Estrategia de la OMS sobre medicina tradicional, 2002-2005. Organización Mundial de la Salud, Ginebra.

- Pierantoni, C. \& Machado, M.(1994) Profesiones de salud: Una formación cuestionada. Educación medica y salud. 28(2): 199-210.

- Pizza, G. (2005) Antonio Gramsci y la Antropología médica contemporánea. Hegemonía, "capacidad de actuar" (agency) y transformaciones de la persona. Revista de Antropología Social de la Universidad Complutense de Madrid 14: 15-32.

- Valverde, C. (2008) Enfermería basada en los significados del paciente: un modelo basado en la narrativa y en la ética del otro. Index de Enfermería. 17(3):157-158.

- Vicedo, T. (2002) Abraham Flexner, pionero de la Educación Médica. Educación Médica Superior. 16(2): $156-163$.

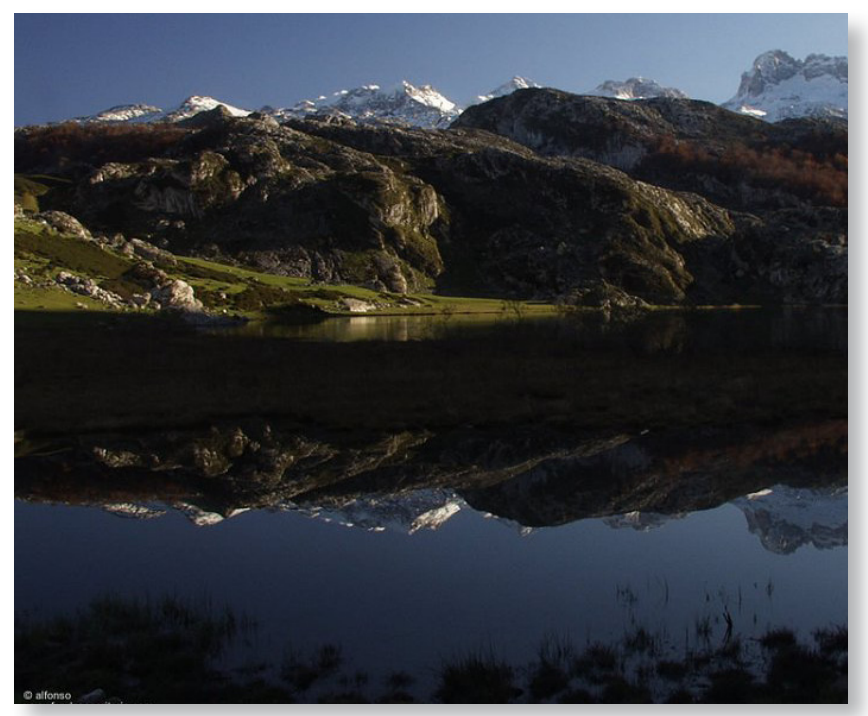

